\title{
EFICIÊNCIA DE ISCAS GRANULADAS (SULFLURAMIDA 0,3\%) NO CONTROLE DE Atta sexdens rubropilosa Forel, 1908 (Hymenoptera: Formicidae)
}

\author{
Efficiency of granulated baits (sulfluramid 0.3\%) in the control of \\ Atta sexdens rubropilosa Forel, 1908 (Hymenoptera: Formicidae)
}

\author{
Ronald Zanetti ${ }^{1}$, Nívia Dias ${ }^{1}$, Marcelo Reis ${ }^{1}$, Alan Souza-Silva ${ }^{1}$, Michelle Alves Moura ${ }^{2}$
}

\begin{abstract}
RESUMO
Foram comparados quatro produtos comerciais à base de sulfluramida (0,3\% i.a.), quanto a sua eficiência no controle de Atta sexdens rubropilosa: Dinagro-S NA, Mirex-S Max, AttaMex-S NA e PikaPau-S NA. Avaliou-se a porcentagem de carregamento e devolução das iscas 24 e 48 horas após a aplicação dos tratamentos. A atividade de corte e movimentação das formigas foi avaliada aos 3, 15, 30, 60, 90, 150, 180 e 210 dias após os tratamentos e a mortalidade dos formigueiros, aos 240 dias. A atividade de forrageamento diminuiu a partir dos três dias após a aplicação, paralisando totalmente as colônias, em média, aos 20,5 $\pm 5,5$ dias. Todos os quatro produtos formicidas foram bem aceitos pelas operárias de A. sexdens rubropilosa e apresentaram igual eficiência no controle de dessa praga, com média de $86,7 \pm 3,2 \%$. O tempo de avaliação operacional da eficiência de 150 dias é suficiente para confirmar a mortalidade dos formigueiros sem a necessidade de escavação total dos ninhos.
\end{abstract}

Termos para indexação: Formigas cortadeiras, controle químico, eucalipto, eficiência de formicidas.

\begin{abstract}
Four granulated baits (sulfluramid $0.3 \%$ a.i.) were compared, in relation to their efficiency in the control of Atta sexdens rubropilosa: Dinagro-S NA, Mirex-S Max, AttaMex-S NA and PikaPau-S NA. Transport and devolution of the baits were evaluated 24 and 48 hours after application. Cutting activity and ant movement were evaluated after 3, 15, 30, 60, 90, 150, 180 and 210 days and mortality after 240 days. Activity of ant nests decreased 3 days after application, paralyzing totally $20.5 \pm$ 5.5 days after application. All products were accepted by the workers of A. sexdens rubropilosa. Efficiencies of these products were $86.7 \pm 3.2 \%$, with no differences among baits and evaluation periods.
\end{abstract}

Index terms: Leaf-cutting ants, chemical control, Eucalyptus, efficiency of baits.

(Recebido para publicação em 28 de julho de 2003 e aprovado em 18 de setembro de 2003)

\section{INTRODUÇÃO}

As formigas cortadeiras dos gêneros Atta e Acromyrmex constituem as principais pragas em reflorestamentos brasileiros (DELLA LUCIA e VILELA, 1993), já que causam prejuízos consideráveis em plantios de Eucalyptus spp., cortando as folhas e ramos tenros, podendo destruir completamente as plantas (OLIVEIRA, 1996).

Dos métodos de controle existentes para formigas cortadeiras, o uso de iscas granuladas tem se destacado (ZANUNCIO et al., 1980), principalmente, por oferecerem maior segurança ao operador (LOECK e NAKANO, 1984), menor custo de mão-de-obra e maior rendimento no campo (CRUZ et al., 1984).

Obtiveram-se bons resultados em experimentos por meio dos quais objetivou-se testar a aceitação de iscas à base de sulfluramida para Atta laevigata F. Smith,
1858, Atta sexdens rubropilosa Forel, 1908, Atta bisphaerica Forel, 1908 e Acromyrmex subterraneus subterraneus Forel, 1893 (DELLA LUCIA et al., 1992), o que possibilitou novos estudos sobre a eficiência desse princípio ativo. Para se testar a eficiência dos diferentes tipos de iscas granuladas existentes no mercado, é de suma importância proceder a testes de aceitação do produto pelas operárias forrageadoras. Os resultados desses ensaios poderão auxiliar na seleção de produtos, visando a reduzir o tempo de aplicação, os custos com mãode-obra e valor de mercado (DELLA LUCIA et al., 1992). Iscas à base de sulfluramida têm se mostrado eficientes no controle das formigas cortadeiras A. laevigata (ZANUNCIO et al., 1992; ALVES et al., 1996), A. bisphaerica (ZANUNCIO et al., 1993) Atta cephalotes L., 1758 (ZANUNCIO et

\footnotetext{
1. Departamento de Entomologia, Universidade Federal de Lavras/UFLA - Caixa Postal 37 - 37200-000 - Lavras, MG. zanetti@ufla.br
}

2. V\&M Florestal Ltda, Praça Voluntários da Pátria, 151 - 35790-000 - Curvelo, MG. michelle@vmtubes.com.br 
al., 1996b), Acromyrmex subterraneus molestans Santschi, 1925 (ZANUNCIO et al., 1996a), A. sexdens rubropilosa (ZANUNCIO, 1997; ZANUNCIO et al., 2002) e Atta sexdens sexdens L., 1758 (CRUZ et al., 1996).

A avaliação experimental da eficiência dessas iscas é feita geralmente entre 120 e 180 dias após a sua aplicação (ZANUNCIO et al., 1999), realizando-se escavações nos formigueiros para comprovar a morte da rainha. A avaliação operacional é realizada geralmente entre 150 e 180 dias, com a raspagem superficial da terra solta e/ou a perfuração de algumas panelas do formigueiro. Todavia, esse tempo para a avaliação operacional pode ser insuficiente para a confirmação da mortalidade dos formigueiros, pois não é feita pela escavação total dos mesmos, devido ao alto custo e maior tempo para essa avaliação.

Neste trabalho, objetivou-se testar a eficiência de quatro iscas granuladas à base de sulfluramida no controle de Atta sexdens rubropilosa (Hymenoptera: Formicidae) em reflorestamentos e estabelecer o período após o combate, para a avaliação operacional da eficiência.

\section{MATERIAL E MÉTODOS}

O experimento foi conduzido em reflorestamentos de eucalipto da empresa V \& M Florestal Ltda., na fazenda Santo Antônio, localizada no município de João Pinheiro, Estado de Minas Gerais.

Foram comparadas quatro marcas comerciais de iscas (Dinagro-S NA, Mirex-S Max, AttaMex-S NA e PikaPau-S NA) à base de sulfluramida $(0,3 \%$ i.a.), quanto à eficiência no controle de Atta sexdens rubropilosa. No teste, foi também incluída uma testemunha que não recebeu isca. Foi utilizado um delineamento inteiramente casualizado com cinco tratamentos (quatro iscas e uma testemunha) e 15 repetições (formigueiros) por tratamentos. Os formigueiros de cada tratamento foram subdivididos em três classes de tamanho: classe I (1-10 $\left.\mathrm{m}^{2}\right)$, classe II (10-30 $\mathrm{m}^{2}$ ), classe III (30-90 $\mathrm{m}^{2}$ de terra solta), contendo cinco formigueiros cada classe. A quantidade de isca fornecida para cada formigueiro foi de $10 \mathrm{~g} / \mathrm{m}^{2}$ de terra solta.

Foram feitas avaliações da porcentagem de carregamento e de devolução da isca 24 e 48 horas após sua aplicação. Também foi avaliada a atividade de forrageamento e de movimentação das formigas aos 3, 15, $30,60,90,150,180,210$ e 240 dias após o tratamento (DAT). A partir dos 150 dias, os formigueiros considerados mortos tiveram sua terra solta raspada e alguns canais perfurados, semelhante ao processo de avaliação operacional de eficiência de controle realizada pelas empresas. Na última avaliação (240 DAT), todos os formigueiros foram escavados totalmente, para avaliação final da eficiência das iscas formicidas, semelhante à avaliação experimental. Foi considerado morto todo formigueiro que não apresentava atividade de formigas nos canais escavados.

Os valores de carregamento e da devolução das iscas e da mortalidade dos formigueiros foram submetidos à análise de variância (teste F) e ao teste de Kruskal-Wallis $(\mathrm{p} \leq 0,05)$. Os valores da paralisação da movimentação das formigas ao longo dos dias de avaliação foram submetidos à análise de variância (teste F) e ao teste de Scott-Knott $(\mathrm{p} \leq 0,05)$.

\section{RESULTADOS E DISCUSSÕES}

Nas avaliações de 24 e 48 horas após a aplicação das iscas, pôde-se constatar que não houve diferenças significativas entre os produtos com relação ao carregamento e à devolução das iscas aplicadas nos formigueiros tratados, inferindo-se que eles apresentam boa aceitação pelas operárias de Atta sexdens rubropilosa (Quadro 1). Esses resultados confirmam aqueles obtidos por Laranjeiro e Zanuncio (1995) e Zanuncio et al. (1996a,b, 1999), que verificaram intenso carregamento dessas iscas por operárias de A. sexdens rubropilosa, Acromyrmex subterraneus molestans, Atta cephalotes e Atta laevigata.

A atividade dos formigueiros tratados com os diferentes produtos formicidas diminuiu a partir dos três dias após a aplicação das iscas. Houve diferença entre os tratamentos, e todos os formicidas causaram paralisação da movimentação das formigas, significativamente superior à testemunha. Não houve diferença significativa entre os dias de avaliação ( $p>0,05)$, demonstrando que o tempo de avaliação operacional da eficiência de 150 dias é suficiente para confirmar a mortalidade dos formigueiros, sem a necessidade de escavação total do ninho (Quadro 2).

Neste trabalho, a paralisação total média da atividade dos formigueiros tratados ocorreu em 20,55 \pm 9,98 dias, enquanto os não-tratados permaneceram com atividade normal (Quadro 3). Della Lucia et al. (1992) observaram que formigueiros tratados com isca á base de dodecacloro paralisam o corte aos nove dias.

Dados sobre o controle de A. sexdens rubropilosa, obtidos em laboratório por Forti et al. (1993), mostraram que os formigueiros tratados com iscas à base de sulfluramida tiveram uma rápida paralisação da atividade de corte das folhas no terceiro e quarto dias após aplicação do produto. Laranjeiro e Zanuncio (1995) obtiveram re- 
sultados semelhantes para a mesma espécie, quando observaram a paralisação total do formigueiro entre o décimo quinto e o trigésimo dia da aplicação da isca.

Zanuncio et al. (1996a), avaliando a eficiência de iscas à base de sulfluramida, verificaram paralisação total da atividade de corte de Acromyrmex subterraneus molestans aos 30 dias, nas dosagens de $6 \mathrm{~g}, 8 \mathrm{~g}$ e 10 $\mathrm{g} / \mathrm{m}^{2}$. Para Atta laevigata, a paralisação ocorreu aos 60 dias com a dosagem de $8 \mathrm{~g} / \mathrm{m}^{2}$ (ZANUNCIO et al., 1999). Para Acromyrmex octospinosus Reich, 1793, aos 15 dias com as dosagens de $8 \mathrm{~g} \mathrm{e} 10 \mathrm{~g} / \mathrm{m}^{2}$ (CRUZ et al., 2000) e para Atta sexdens rubropilosa, aos 10 dias com a dosagem de $10 \mathrm{~g} / \mathrm{m}^{2}$ (ZANUNCIO et al., 2002).

QUADRO 1 - Porcentagem média de carregamento e de devolução após 24 e 48 horas respectivamente, da aplicação das iscas nos formigueiros de Atta sexdens rubropilosa.

\begin{tabular}{|c|c|c|}
\hline Tratamento & Carregamento após 24 horas & Devolução após 48 horas \\
\hline Dinagro-S NA & 94,33 & 6,00 \\
PikaPau-S NA & 95,67 & 3,33 \\
Mirex-S Max & 72,67 & 8,33 \\
AttaMex-S NA & 81,06 & 2,81 \\
\hline
\end{tabular}

${ }^{1}$ Os tratamentos não diferiram entre si $(\mathrm{F} ; \mathrm{p}>0,05)$.

QUADRO 2 - Paralisação da atividade dos formigueiros (\%) de Atta sexdens rubropilosa em diferentes períodos após a aplicação das iscas.

\begin{tabular}{|c|c|c|c|c|c|c|c|c|c|c|}
\hline \multirow{2}{*}{ Tratamento } & \multicolumn{8}{|c|}{ Período de avaliação (DAT)* } & \multirow{2}{*}{ Médias ${ }^{1}$} \\
\cline { 2 - 10 } & $\mathbf{3}$ & $\mathbf{1 5}$ & $\mathbf{3 0}$ & $\mathbf{6 0}$ & $\mathbf{9 0}$ & $\mathbf{1 5 0}$ & $\mathbf{1 8 0}$ & $\mathbf{2 1 0}$ & $\mathbf{2 4 0}$ & \\
\hline Testemunha & 0,0 & 0,0 & 0,0 & 0,0 & 0,0 & 0,0 & 0,0 & 0,0 & 0,0 & $0,0 \mathrm{~b}$ \\
PikaPau-S NA & 93,3 & 93,3 & 93,3 & 93,3 & 86,6 & 93,3 & 93,3 & 86,6 & 86,6 & 91,1 a \\
AttaMex-S NA & 93,3 & 86,6 & 93,3 & 86,6 & 93,3 & 86,6 & 86,6 & 80,0 & 73,3 & 86,6 a \\
Dinagro-S NA & 93,3 & 86,6 & 100,0 & 100,0 & 100,0 & 100,0 & 100,0 & 100,0 & 100,0 & 97,7 a \\
Mirex-S Max & 100,0 & 93,3 & 100,0 & 93,3 & 93,3 & 93,3 & 93,3 & 86,6 & 86,6 & 93,3 a \\
\hline Médias & $76,0 \mathrm{~A}$ & $72,0 \mathrm{~A}$ & $77,3 \mathrm{~A}$ & $74, \mathrm{~A}$ & $74,6 \mathrm{~A}$ & $74,6 \mathrm{~A}$ & $74,6 \mathrm{~A}$ & $70,6 \mathrm{~A}$ & $69,3 \mathrm{~A}$ & \\
\hline
\end{tabular}

*Médias seguidas de mesma letra minúscula na coluna e maiúscula na linha são estatisticamente iguais (Scott-Knott; $\mathbf{p}>\mathbf{0 , 0 5}$ ). 
QUADRO 3 - Tempo médio decorrido para paralisação total da atividade de formigueiros de Atta sexdens rubropilosa e porcentagem de mortalidade dos formigueiros aos 240 dias após a aplicação dos tratamentos.

\begin{tabular}{|c|c|c|}
\hline Tratamento & $\begin{array}{c}\text { Tempo para paralisação } \\
\text { atividade (dias) }\end{array}$ & da \\
\hline Dinagro-S NA & $7,4 \pm 2,5 \mathrm{~b}$ & Mortalidade (\%) \\
\hline PikaPau-S NA & $29,2 \pm 13,9 \mathrm{~b}$ & $87,0 \pm 6,6 \mathrm{~b}$ \\
Mirex-S Max & $14,6 \pm 9,8 \mathrm{~b}$ & $87,0 \pm 6,6 \mathrm{~b}$ \\
AttaMex-S NA & $31,0 \pm 13,7 \mathrm{~b}$ & $73,0 \pm 8,5 \mathrm{~b}$ \\
Testemunha & $0,0 \pm 0,0 \mathrm{a}$ & $0,0 \pm 0,0 \mathrm{a}$ \\
\hline TMPT** & $20,55 \pm 9,98$ & - \\
\hline
\end{tabular}

*Médias dos tratamentos seguidas pela mesma letra são estatisticamente iguais (Kruskal-Wallis; $\mathbf{p}>\mathbf{0 , 0 5})$. ** tempo médio para paralisação nos tratamentos com iscas.

Não houve diferença significativa na mortalidade dos formigueiros tratados com os produtos, sendo a porcentagem média de mortalidade dos formigueiros tratados de 86,7 $\pm 3,2$ dias (Quadro 3). Assim, todas as iscas formicidas testadas foram eficientes, podendo ser recomendadas indistintamente. Esses resultados estão de acordo com os observados por Zanuncio et al. (2002), que avaliaram a eficiência dessa isca contra $A$. sexdens rubropilosa, e constataram que, aos 150 dias, a isca à base de sulfluramida apresentou eficiência de $90 \%$.

\section{CONCLUSÕES}

Todas as iscas formicidas testadas apresentam igual eficiência no controle de Atta sexdens rubropilosa. O tempo de avaliação operacional da eficiência de 150 dias é suficiente para confirmar a mortalidade dos formigueiros, sem a necessidade de escavação total dos ninhos.

\section{AGRADECIMENTOS}

À V \& M Florestal Ltda, pela concessão da área e pelo apoio de campo. À Basf S.A - Departamento de Saúde Ambiental e à FAPEMIG, pelo auxílio financeiro.

\section{REFERÊNCIAS BIBLIOGRÁFICAS}

ALVES, J. B.; ZANUNCIO, J. C.; TORRES, J. B. Métodos de distribuição de isca granulada em formigueiros de Atta laevigata (F. SMITH). Revista Árvore, Viçosa, v. 20, n. 1, p. 111-116, jan./mar. 1996.
CRUZ, A. P.; ZANUNCIO, J. C.; ZANETTI, R. Eficiência de iscas granulada à base de sulfluramida e de clorpirifós no controle de Atta sexdens sexdens (Hymenoptera: Formicidae), no trópico úmido. Acta Amazônica, Manaus, v. 26, n. 3, p. 145-150, jul. 1996.

CRUZ, A. P.; ZANUNCIO, J. C.; ZANETTI, R. Eficiência de cebos granulados a base de sulfluramida o de clorpirifós em el control de Acromyrmex octospinosus (Hymenoptera: Formicidae) em el trópico húmedo. Revista Colombiana de Entomología, Bogotá, v. 26, n. 1-3, p. 67-69, ene./jun. 2000.

CRUZ, J. M.; NOGUEIRA, S. B.; PEREIRA, A. R. Adaptação de uma motocicleta para termonebulização no controle de formigas saúvas (Atta spp.), em áreas reflorestadas de cerrado. Revista Árvore, Viçosa, v. 8, n. 2, p. 104-111, jul./dez. 1984.

DELlA LUCIA, T. M. C.; CAMERON, R. S.; VILELA, E. F. Aceitação de iscas granuladas com sulfluramida, um novo princípio ativo, por formigas cortadeiras, no campo. Revista Árvore, Viçosa, v. 16, n. 2, p. 218-223, maio/ago. 1992.

DELLA LUCIA, T. M. C.; VILELA, E. V. Métodos atuais de controle e perspectivas. In: DELLA LÚCIA, T. M. C. As formigas cortadeiras. Viçosa: Folha de Viçosa, 1993. p. 163-190. 
FORTI, L. C.; PINHÃO, M. A. S.; YASSU, W. K. Pesquisas com sulfluramida, no período de 1989 a 1993, para o controle de Atta spp. In: INTERNATIONAL PEST ANT SYMPOSIUM, 4.; ENCONTRO DE MIRMECOLOGIA, 11., 1993, Belo Horizonte. Anais... Belo Horizonte: [s.n.], 1993. p. 23.

LARANJEIRO, A. J.; ZANUNCIO, J. C. Avaliação da isca à base de sulfluramida no controle de Atta sexdens rubropilosa pelo processo de dosagem única de aplicação. IPEF, Piracicaba, v. 48, n. 49, p. 144-152, jun./dez. 1995.

LOECK, A. E.; NAKANO, O. Efeito de novas substâncias visando o controle de sauveiros novos de Atta laevigata (Smith, 1858) (Hymenoptera: Formicidae). 0 Solo, Piracicaba, v. 76, n. 1, p. 25-30, jan./jun. 1984.

OLIVEIRA, M. A. Identificação de formigas cortadeiras e efeito do desfolhamento simulado em plantios de Eucalyptus grandis. 1996. 61 f. Dissertação (Mestrado em Entomologia) - Universidade Federal de Viçosa, Viçosa, 1996.

ZANUNCIO, J. C. Controle de Atta laevigata e Atta sexdens rubropilosa (F. Smith, 1858) com as iscas granuladas Mirex-S Max e Mirex-S Plus. In INTERNATIONAL PEST ANT SYMPOSIUM, 6.; ENCONTRO DE MIRMECOLOGIA, 13., 1997, Ilhéus. Anais... Ilhéus: [s.n.], 1997. p. 163.

ZANUNCIO, J. C.; COUTO, L.; SANTOS, G. P. Eficiência da isca granulada Mirex-S, à base de sulfluramida, no controle da formiga cortadeira Atta laevigata ( $\mathrm{F}$. SMITH, 1858) (Hymenoptera: Formiciae). Revista Árvore, Viçosa, v. 16, n. 3, p. 357-361, set./dez. 1992.
ZANUNCIO, J. C.; COUTO, L.; ZANUNCIO, T. V. Eficiência da isca granulada Mirex-S, (sulfluramida 0,3\%) no controle da formiga-cortadeira Atta bisphaerica FOREL (Hymenoptera: Formiciae). Revista Árvore, Viçosa, v. 17, n. 1, p. 85-90, jan./mar. 1993.

ZANUNCIO, J. C.; CRUZ, A. P.; SANTOS, D. F. Eficiência da isca Mirex-S (sulfluramida $0,3 \%$ ) no controle de Atta cephalotes (Hymenoptera: Formiciae) em três dosagens. Acta Amazônica, Manaus, v. 26, n. 1/2, p. 115-120, nov. 1996 b.

ZANUNCIO, J. C.; LARANJEIRO, A. J. Controle de Acromyrmex subterraneus molestans Santschi (Hymenoptera: Formicidae) com sulfluramida. Anais da Sociedade Entomológica do Brasil, Londrina, v. 25, n. 3, p. 383-388, dez. 1996 a.

ZANUNCIO, J. C.; SOSSAI, M. F.; OLIVEIRA, H. $\mathrm{N}$. Influência das iscas formicidas Mirex-S Max e Blitz na paralisação de corte e no controle de Atta sexdens rubropilosa (Hymenoptera: Formicidae). Revista Árvore, Viçosa, v. 26, n. 2, p. 237-242, mar./abr. 2002.

ZANUNCIO, J. C.; VILELA, E. F.; NOGUEIRA, S. B. Emprego de iscas granuladas e pós secos no controle de Atta laevigata, no município de Curvelo. Revista Árvore, Viçosa, v. 4, n. 2, p. 221-226, jul./dez. 1980.

ZANUNCIO, J. C.; ZANUNCIO, T. V.; PEREIRA, J. M. M. Controle de Atta laevigata (Hymenoptera: Formiciae) com a isca Landrin-F, em área anteriormente coberta com Eucalyptus. Ciência Rural, Santa Maria, v. 29, n. 4 , p. 573-576, jul./set. 1999. 